\title{
Continuum QRPA response for deformed neutron-rich nuclei
}

\author{
K. Hagino ${ }^{\mathrm{a}}$, Nguyen Van Giai ${ }^{\mathrm{b}}$, H. Sagawa ${ }^{\mathrm{c}}$ \\ ${ }^{a}$ Yukawa Institute for Theoretical Physics, Kyoto University, Kyoto 606-8502, Japan \\ bInstitut de Physique Nucléaire, IN2P3-CNRS, Université Paris-Sud, F-91406 Orsay \\ Cedex, France \\ ${ }^{\mathrm{c}}$ Center for Mathematical Sciences, University of Aizu, Aizu-Wakamatsu, Fukushima \\ 965-8560, Japan
}

We discuss properties of the quadrupole collective excitation of the deformed neutronrich nucleus ${ }^{38} \mathrm{Mg}$ within the framework of quasi-particle random phase approximation (QRPA). We first solve the coupled-channels equations to obtain the single-particle levels, and construct the ground state by treating the pairing correlations in the BCS approximation. We then solve the QRPA equation using the response function formalism, by including the continuum spectra with the box dicscretization method. We show that the collectivity of the gamma vibration (the lowest $K^{\pi}=2^{+}$mode) is significantly enhanced if protons and neutrons have different deformations. We also discuss an attempt towards full continuum QRPA calculations for deformed nuclei.

\section{Introduction}

Without an exception, stable nuclei with a neutron number $N=20$ or 28 , as well as their neigbouring nuclei, have a spherical intrinsic shape. Heavy neutron-rich nuclei, however, often exhibit deformed characters even around the shell closure. Typical examples include ${ }^{32} \mathrm{Mg}$ [ 1], ${ }^{34} \mathrm{Mg}$ [ 2, 3], and ${ }^{44} \mathrm{~S}$ [ 4]. This phenomenon is often interpreted as a disappearance of spherical magic shells [ [5, 6].

In this contribution, we investigate the excitation structures of such deformed neutronrich nuclei within the framework of quasi-particle random phase approximation (QRPA). For nuclei far from the $\beta$ stability line, it has been well recognised that both the pairing interaction and the couplings to the continuum spectra play an important role. The most robust and consistent framework to deal with these effects on the ground state properties is the Hartree-Fock-Bogoliubov (HFB) method [ 7, 8]. The continuum QRPA framework based on the HFB approximation has also been developed recently [ 9, 10]. A problem with the HFB method, however, is that it can be computationally very demanding. For this reason, the application of the continuum QRPA on top of the HFB ground state has been limited only to spherical systems so far [9, 10]. In this paper, we avoid this difficulty by using the BCS approximation. This is an approximation to the HFB method, where one takes only time-reversed pairs in the Bogoliubov transformation. With this approximation, the particle-hole and the particle-particle channels are decoupled completely (for 
a density-independent pairing force at least) both in the ground state and in the QRPA calculations. Notice that the BCS method has been shown a reasonable approximation to the HFB, within the same treatment for the continuum states, even for nuclei close to the neutron drip line [8, 11].

Another difficulty related to a deformed QRPA calculation is that the configuration space can be quite large. In the past, QRPA calculations for a deformed nucleus have been done either with a simple separable interaction [12] or with a drastic truncation of the configuration space [13]. In this paper, we present a formalism which uses the response function in the coordinate space [14, which is most suitable to a zero-range interaction, eliminating these limitations. Together with the BCS approximation for the pairing interaction, we will demonstrate below that a deformed QRPA calculation now becomes feasible within a reasonable computation time.

\section{Formalism}

\subsection{Coupled-channels approach to single-particle spectra}

Let us consider a single-particle motion in a deformed mean-field potential,

$\hat{V}(\boldsymbol{r})=V_{\text {cent }}(\boldsymbol{r})+\nabla\left(V_{\mathrm{ls}}(\boldsymbol{r})\right) \cdot(-i \nabla \times \boldsymbol{\sigma})$,

with

$$
\begin{aligned}
V_{\text {cent }}(\boldsymbol{r}) & =V_{0}(r)-R \beta \frac{d V_{0}(r)}{d r} Y_{20}(\hat{\boldsymbol{r}})+\left(V_{C}(r)+\frac{3(Z-1)}{5} \frac{R^{2}}{r^{3}} \beta Y_{20}(\hat{\boldsymbol{r}})\right)\left(1-\tau_{z}\right), \\
V_{\mathrm{ls}}(\boldsymbol{r}) & =V_{\mathrm{so}}(r)-R_{\mathrm{so}} \beta \frac{d V_{\mathrm{so}}(r)}{d r} Y_{20}(\hat{\boldsymbol{r}})
\end{aligned}
$$

Here, $\beta$ is the deformation parameter. For simplicity, we take into account only the linear order of $\beta$ in the potential. We have also assumed an axial symmetry, where both the parity $\pi$ and the spin projection $K$ onto the $z$ axis are conserved.

In order to obtain the single-particle levels and corresponding wave functions, we solve the coupled-channels equations for both negative and positive energy states [15]. This method has an advantage that the correct scattering boundary conditions can be easily implemented for the positive energy solutions, that is important in a full continuum calculation for neutron-rich nuclei. In this paper, for the sake of simplicity, we instead discretize the positive energy spectra within the same framework by putting the nucleus in a large spherical box, as was recently demonstrated in Ref. [15], and leave the full continuum treatment as a future work (see sect. 4).

The coupled-channels method has sometimes a difficulty in finding all solutions in a deformed potential, especially when there are two states close to each other in energy. One can easily miss a few eigenvalues in a search routine. We have overcome this difficulty by introducing the generalized wave function node for a multi-channel system, which was originally proposed by Johnson [16]. We have checked the efficiency of this method by applying it to a spherical system. We have also computed the Nilsson diagram of ${ }^{238} \mathrm{U}$ and have confirmed that all the levels obtained were smoothly connected to the spherical levels. Evidently, the coupled-channels method with the generalized wave function node efficiently works even for heavy nuclei, where there are lots of intruder levels. 
Using the single-particle levels obtained in this way, we then solve the BCS gap equation. As we emphasized in the introduction, we use the BCS approximation rather than the HFB approximation. The applicability of the BCS approximation for neutron-rich nuclei has been well tested in Ref. [ 8, 11]. In this paper, we particularly use the densitydependent zero-range force for the pairing interaction. We will specify the parameters in the next section.

\subsection{Response function formalism for deformed QRPA}

The elements of the QRPA matrices have been given in the textbook of Rowe [17]:

$$
\begin{aligned}
A_{\alpha \beta, \gamma \delta}= & \left(E_{\alpha}+E_{\beta}\right) \delta_{\alpha \beta, \gamma \delta}+\left\langle\alpha \bar{\delta}\left|v_{p h}\right| \bar{\beta} \gamma\right\rangle\left(u_{\alpha} v_{\beta}+v_{\alpha} u_{\beta}\right)\left(u_{\gamma} v_{\delta}+v_{\gamma} u_{\delta}\right) \\
& +\left\langle\alpha \beta\left|v_{p p}\right| \gamma \delta\right\rangle\left(u_{\alpha} u_{\beta} u_{\gamma} u_{\delta}+v_{\alpha} v_{\beta} v_{\gamma} v_{\delta}\right) \\
B_{\alpha \beta, \gamma \delta}= & \left\langle\alpha \gamma\left|v_{p h}\right| \bar{\beta} \bar{\delta}\right\rangle\left(u_{\alpha} v_{\beta}+v_{\alpha} u_{\beta}\right)\left(u_{\gamma} v_{\delta}+v_{\gamma} u_{\delta}\right) \\
& -\left\langle\alpha \beta\left|v_{p p}\right| \bar{\gamma} \bar{\delta}\right\rangle\left(u_{\alpha} u_{\beta} v_{\gamma} v_{\delta}+v_{\alpha} v_{\beta} u_{\gamma} u_{\delta}\right)
\end{aligned}
$$

where $E_{\alpha}$ is the quasi-particle energy and $\bar{\alpha}$ is the time reversed state of $\alpha$. In writing down these equations, we have used the identity, $\langle\alpha \bar{\delta}|v| \bar{\beta} \gamma\rangle=-\langle\alpha \bar{\gamma}|v| \bar{\beta} \delta\rangle$, which follows from the property of the time-reversal operator. Notice that the particle-particle matrix elements vanish in the BCS approximation unless $\beta=\bar{\alpha}$ and $\delta=\bar{\gamma}$ in eqs. (4) and (5). On the other hand, the particle-hole contribution is finite only if $\beta \neq \bar{\alpha}$ and $\delta \neq \bar{\gamma}$ due to the BCS phase convention, $v_{\bar{\alpha}}=-v_{\alpha}$ and $u_{\bar{\alpha}}=u_{\alpha}$. Therefore, the particle-particle and the particle-hole channels are decoupled completely in the BCS approximation, not only in the ground state but also in the QRPA calculations.

Retaining only the particle-hole channel, one can write down the QRPA equations in the following form:

$$
\begin{aligned}
& 0=\left(E_{\alpha}+E_{\beta}-\omega\right) X_{\alpha \beta}+\int d \boldsymbol{r} d \boldsymbol{r}^{\prime} D_{\alpha \bar{\beta}}(\boldsymbol{r}) v_{p h}\left(\boldsymbol{r}, \boldsymbol{r}^{\prime}\right) T\left(\boldsymbol{r}^{\prime}\right), \\
& 0=\left(E_{\alpha}+E_{\beta}+\omega\right) Y_{\alpha \beta}+\int d \boldsymbol{r} d \boldsymbol{r}^{\prime} D_{\alpha \bar{\beta}}^{*}(\boldsymbol{r}) v_{p h}\left(\boldsymbol{r}, \boldsymbol{r}^{\prime}\right) T\left(\boldsymbol{r}^{\prime}\right) .
\end{aligned}
$$

Here, we have explicitly expressed the matrix elements of the particle-hole interaction in the integral form. Using the single-particle wave function $\phi_{\alpha}(\boldsymbol{r}), D_{\alpha \bar{\beta}}(\boldsymbol{r})$ and $T(\boldsymbol{r})$ are defined by

$$
\begin{aligned}
D_{\alpha \bar{\beta}}(\boldsymbol{r}) & =\left(u_{\alpha} v_{\beta}+v_{\alpha} u_{\beta}\right) \phi_{\alpha}^{*}(\boldsymbol{r}) \phi_{\bar{\beta}}(\boldsymbol{r}), \\
T(\boldsymbol{r}) & =\sum_{\gamma \delta}\left(D_{\gamma \bar{\delta}}^{*}(\boldsymbol{r}) X_{\gamma \delta}+D_{\gamma \bar{\delta}}(\boldsymbol{r}) Y_{\gamma \delta}\right),
\end{aligned}
$$

respectively. From these equations, one obtains the generalised (Q)RPA dispersion relation [18],

$0=\int d \boldsymbol{r}^{\prime}\left[\delta\left(\boldsymbol{r}-\boldsymbol{r}^{\prime}\right)-\int d \boldsymbol{r}^{\prime \prime} \Pi_{0}\left(\boldsymbol{r}, \boldsymbol{r}^{\prime \prime}\right) v_{p h}\left(\boldsymbol{r}^{\prime \prime}, \boldsymbol{r}^{\prime}\right)\right] T\left(\boldsymbol{r}^{\prime}\right) \equiv \int d \boldsymbol{r}^{\prime} W\left(\boldsymbol{r}, \boldsymbol{r}^{\prime}\right) T\left(\boldsymbol{r}^{\prime}\right)$,

where the unperturbed response function is given by

$\Pi_{0}\left(\boldsymbol{r}, \boldsymbol{r}^{\prime \prime}\right)=-\sum_{\alpha \beta}\left(\frac{D_{\alpha \bar{\beta}}^{*}(\boldsymbol{r}) D_{\alpha \bar{\beta}}\left(\boldsymbol{r}^{\prime}\right)}{E_{\alpha}+E_{\beta}-\omega-i \eta}+\frac{D_{\alpha \bar{\beta}}(\boldsymbol{r}) D_{\alpha \bar{\beta}}^{*}\left(\boldsymbol{r}^{\prime}\right)}{E_{\alpha}+E_{\beta}+\omega+i \eta}\right)$. 
The response of the system to an external field can be calculated using the QRPA response function defined by [14]

$\Pi\left(\boldsymbol{r}, \boldsymbol{r}^{\prime}\right)=\int d \boldsymbol{r}^{\prime \prime} W^{-1}\left(\boldsymbol{r}, \boldsymbol{r}^{\prime \prime}\right) \Pi_{0}\left(\boldsymbol{r}^{\prime \prime}, \boldsymbol{r}^{\prime}\right)$.

For axially symmetric wave functions $\phi_{\alpha}$, the response function can be decomposed into multipoles as

$\Pi\left(\boldsymbol{r}, \boldsymbol{r}^{\prime}\right)=\sum_{L, L^{\prime}} \sum_{K} \frac{1}{r r^{\prime}} \Pi_{K}\left(r L, r^{\prime} L^{\prime}\right) Y_{L K}(\hat{\boldsymbol{r}}) Y_{L^{\prime} K}^{*}\left(\hat{\boldsymbol{r}}^{\prime}\right)$.

We assume a zero range particle-hole interaction of the form

$v_{p h}\left(\boldsymbol{r}, \boldsymbol{r}^{\prime}\right)=\left[\sum_{\lambda: \text { even }} v_{\lambda}(r) Y_{\lambda 0}(\hat{\boldsymbol{r}})\right] \delta\left(\boldsymbol{r}-\boldsymbol{r}^{\prime}\right)$.

Then, the QRPA response function (12) is obtained by solving the Bethe-Salpeter equation for each $K$,

$\Pi_{K}\left(r L, r^{\prime} L^{\prime}\right)=\Pi_{K}^{(0)}\left(r L, r^{\prime} L^{\prime}\right)+\sum_{L^{\prime \prime}} \int d r^{\prime \prime}\left[\Pi^{(0)} v\right]_{K}\left(r L, r^{\prime \prime} L^{\prime \prime}\right) \Pi_{K}\left(r^{\prime \prime} L^{\prime \prime}, r^{\prime} L^{\prime}\right)$,

where

$$
\left[\Pi^{(0)} v\right]_{K}\left(r L, r^{\prime} L^{\prime}\right) \equiv \sum_{\lambda} \sum_{L^{\prime \prime}} \Pi_{K}^{(0)}\left(r L, r^{\prime} L^{\prime \prime}\right) v_{\lambda}\left(r^{\prime}\right)\left\langle Y_{L^{\prime \prime} K}\left|Y_{\lambda 0}\right| Y_{L^{\prime} K}\right\rangle .
$$

The strength function $S$ for an external field $V_{\text {ext }}(\boldsymbol{r})=f(r) Y_{L K}(\hat{\boldsymbol{r}})$ is then obtained in the usual way as,

$$
\begin{aligned}
S(\omega) & =-\frac{1}{\pi} \operatorname{Im} \int d \boldsymbol{r} d \boldsymbol{r}^{\prime} V_{\mathrm{ext}}^{*}(\boldsymbol{r}) \Pi\left(\boldsymbol{r}, \boldsymbol{r}^{\prime}\right) V_{\mathrm{ext}}\left(\boldsymbol{r}^{\prime}\right) \\
& =-\frac{1}{\pi} \operatorname{Im} \int r^{2} d r r^{\prime 2} d r^{\prime} \frac{1}{r r^{\prime}} \Pi_{K}\left(r L, r^{\prime} L\right) f(r) f\left(r^{\prime}\right),
\end{aligned}
$$

where $I m$ denotes the imaginary part.

A typical dimensionality of the QRPA response function $\Pi_{K}$ goes as follows. If one includes the radial mesh up to $r=15 \mathrm{fm}$ with $0.5 \mathrm{fm}$ step, there are 30 points for the $r$ integrals. For the interaction given by eq. (14), only those $L$ with the same parity are coupled to each other, and generally the convergence is rather quick. Therefore, for the quadrupole response for example, one would include only $L=0,2$ and 4 . By treating proton and neutron separately, the total dimension of the response function is 30 (for $r$ ) $\times 3$ (for $L) \times 2\left(\right.$ for $\left.\tau_{z}\right)=180$. This is much smaller than the number of 2 quasi-particle configurations, which can be more than several thousands for a deformed system.

\section{Quadrupole collectivity of ${ }^{38} \mathrm{Mg}$}

We now apply the formalism to the quadrupole response of the ${ }^{38} \mathrm{Mg}$ nucleus. We assume a Woods-Saxon form for the mean-field potential $V_{0}(r)$ and $V_{\text {so }}(r)$ in eqs. (2) and (32) with parameters given in Ref. [19]. We take the deformation parameters $\beta$ from the 
results of HFB calculations with a Skyrme interaction by Terasaki et al. [ 20]. For ${ }^{38} \mathrm{Mg}$, the deformation parameters are 0.33 and 0.28 for protons and neutrons, respectively. Notice that the deformation parameters are different for protons and neutrons by an amount of 0.05. We use the same pairing interaction as in Ref. [ 20] for our BCS calculations. This is a density-dependent zero-range interaction with an energy cut-off at $5 \mathrm{MeV}$ above the Fermi energy. Although a smooth energy cut-off was employed in Ref. [20], we use a sharp energy cut-off for simplicity. With these parameters, we obtain $\lambda_{p}=-24.4 \mathrm{MeV}$ and $\lambda_{n}=-0.56 \mathrm{MeV}$ for the proton and the neutron Fermi energies, $\bar{\Delta}_{p}=0.0 \mathrm{MeV}$ and $\bar{\Delta}_{n}=1.50 \mathrm{MeV}$ for the proton and the neutron average pairing gaps. For the residual interaction $v_{p h}$, we use the SIII parameter set [21] of the Skyrme interaction with the Landau-Migdal approximation for the momentum dependent terms [ 18. We include the single-particle levels up to $50 \mathrm{MeV}$ in the calculations. The continuum states are discretized with the box discretization method with the box size of $15 \mathrm{fm}$. We have checked that the results are not altered by more than $10 \%$ even if we use a larger box size. For single-particle states above the pairing cut-off energy, we simply take $v^{2}=0$ for the BCS occupation probabilities.

Since our calculations are not self-consistent in the sense that the residual interaction is not related to the mean field potential, we renormalize the residual interaction as $f \cdot v_{p h}$ so that the Goldstone modes appear at zero excitation energy. For a spherical system, there is only one Goldstone mode for the center-of-mass motion, and one can use a single value for the renormalization factor in order to define the effective residual interaction and study several multipole responses. In contrast, for a deformed system, the center-of-mass motion splits into two excitation modes according to the $K$ quantum number $\left(K^{\pi}=0^{-}\right.$ and $\left.1^{-}\right)$. Moreover, there is another Goldstone mode associated with the rotation motion of the whole nucleus. This motion carries $K^{\pi}=1^{+}$. We find that the renormalization factors required to put these modes at zero energy are significantly different from each other for the configuration space mentioned above. The renormalization factors are found to be 1.00075 for $K^{\pi}=0^{-}, 0.95585$ for $K^{\pi}=1^{-}$, and 1.0300 for $K^{\pi}=1^{+}$. Since we are interested in only the quadrupole motion in this paper, our prescription is to use $f_{1^{+}}$for the other $K$ quantum numbers, i.e., $K^{\pi}=0^{+}$and $2^{+}$. Note that the Goldstone mode for the number conservation does not appear in our calculation, since we include only the particle-hole channel in QRPA.

Figure 1 shows the isoscaler (IS) quadrupole strength distribution of the ${ }^{38} \mathrm{Mg}$ nucleus (the lower panel) in comparison with that of the ${ }^{24} \mathrm{Mg}$ nucleus (the upper panel). We set $\eta=0.5 \mathrm{MeV}$ in eq. (11) in order to smear out the results for the presentation purpose. The dotted, dashed, and solid lines are for $K^{\pi}=0^{+}, 1^{+}$, and $2^{+}$modes, respectively. Since we use a density-dependent pairing interaction, there is a small coupling between the particle-hole and the particle-particle channels for the $K^{\pi}=0^{+}$mode (notice that a timereversed pair can form only a $K^{\pi}=0^{+}$state) even in the BCS approximation, when the residual interaction is evaluated as the second derivative of the energy functional [ 9, 10]. We simply neglect this effect in this work. The $K^{\pi}=1^{+}$and $2^{+}$modes are free from this approximation.

In the figure, one observes the splitting of the giant quadrupole resonances (GQR) [ 22$]$ in the energy region $10-20 \mathrm{MeV}$ both in ${ }^{24} \mathrm{Mg}$ and in ${ }^{38} \mathrm{Mg}$. In contrast to the stable nucleus ${ }^{24} \mathrm{Mg}$, we notice that the IS GQR is more fragmented in the neutron-rich nucleus 
${ }^{38} \mathrm{Mg}$ due to a large neutron contribution. We have also computed the neutron response, and found that the $K^{\pi}=2^{+}$mode in the energy region $3-10 \mathrm{MeV}$ is almost a pure neutron mode. Another interesting result is that the collectivity of the lowest $K^{\pi}=2^{+}$ mode is increased considerably in ${ }^{38} \mathrm{Mg}$. This is due both to the pairing effect [14] and to the different deformation between proton and neutron, as we discuss below.

In Ref. [20], Terasaki et al. have suggested that a low-lying quadrupole isovector mode may appear when the protons and the neutrons have different deformation parameters. In neutron-rich nuclei, the isovector and the isoscalar modes are coupled to each other [ 23. and thus this effect would also enhance the low-lying isoscalar quadrupole strength. In order to confirm this, we repeat the calculations by setting the proton and the neutron deformation parameters to be identical. Figure 2 shows the low-lying part of the isoscalar quadrupole response of ${ }^{38} \mathrm{Mg}$ for the $K^{\pi}=0^{+}$(the top panel), $K^{\pi}=1^{+}$(the middle panel), and $K^{\pi}=2^{+}$(the bottom panel). The dotted and the dashed curves are for the case where the proton and the neutron deformations are set to be the smaller value 0.28 and the larger value 0.33 , respectively. The solid line denotes the original calculation shown in fig. 1, where the proton and the neutron have different deformations. For $K^{\pi}=0^{+}$and $1^{+}$, those three lines are very similar to each other, while, in marked contrast, the lowest $K^{\pi}=2^{+}$excitation (the gamma vibration) clearly shows a different behaviour. When the deformation is different between proton and neutron, the excitation energy of this stae becomes significantly smaller and at the same time the strength is increased considerably. The effect of a larger proton deformation parameter simply changes the dotted curve to the dashed curve. There is an additional effect originating from the different proton and neutron deformations, that enhances the collectivity of the gamma vibration.

\section{Concluding remarks}

At present, a continuum QRPA calculation based on the HFB method is still too costly to apply to deformed nuclei. The only possible way to overcome this difficulty is to employ the BCS approximation for the pairing correlations, where the particle-hole and the particle-particle channels are decoupled. Contrary to a general belief, the BCS approximation for the description of ground state properties has been shown adequate even for neutron-rich nuclei [ 8, 11]. We presume that this is the case also for the description of excited states, and have developed the QRPA method based on the BCS approximation. Particularly, we have presented the response function method for deformed QRPA, which substantially reduces the computational effort to solve QRPA compared with the conventional method which diagonalises the non-hermitian QRPA matrix. We have applied the method to quadrupole responses of the deformed neutron-rich nucleus ${ }^{38} \mathrm{Mg}$. We have shown that the collectivity of the gamma vibration is increased when the protons and the neutrons deform in a different way from each other as the mean-field calculation predicts.

In this paper, we have discretized the continuum states by putting the nucleus in a large spherical box. For a more consistent description, one would need to treat the continuum spectra exactly. This applies both to the BCS and to the QRPA calculations. In the continuum BCS calculation for the ground state, the single-particle resonance states play an essential role [11]. Recently, we have shown that the coupled-channels approach with the eigenchannel basis offers an efficient way to identify a deformed single- 
particle resonance state through the so called eigenphase sum [15]. We have also derived a factorization formula for the energy and the radial dependences of a scattering wave function near an isolated resonance state [ 15]. This formula will be useful when one computes the matrix elements of pairing interaction over a wide energy region including the continuum spectra. As for a continuum QRPA calculation, it is well known that the exact treatment of the continuum couplings is possible if one uses the single-particle Green function in the coordinate space representation [24]. An extension of this approach to a deformed system has been done in the field of atomic and molecular physics [ 25], which again uses the coupled-channels method with the eigenchannel basis (see also Ref. [ [26]). We have also recently extended the Green function approach to spherical open-shell nuclei within the BCS approximation [14]. We plan to combine these two extensions of the Green function approach in order to calculate the continuum response of superfluid deformed nuclei built on top of the continuum BCS ground state. We will report the results elsewhere.

\section{REFERENCES}

1. T. Motobayashi, Eur. Phys. J. A15 (2002) 99.

2. K. Yoneda et al., Phys. Lett. B499 (2001) 233.

3. H. Iwasaki et al., Phys. Lett. B522 (2001) 227.

4. D. Sohler et al., Phys. Rev. C66 (2002) 054302.

5. E.K. Warburton, J.A. Becker, and B.A. Brown, Phys. Rev. C41 (1990) 1147.

6. T. Otsuka et al., Phys. Rev. Lett. 87 (2001) 082502.

7. J. Dobaczewski et al., Phys. Rev. C53 (1996) 2809.

8. M. Grasso et al., Phys. Rev. C64 (2001) 064321.

9. M. Matsuo, Nucl. Phys. A696 (2001) 371.

10. E. Khan et al., Phys. Rev. C66 (2002) 024309.

11. N. Sandulescu, Nguyen Van Giai, and R.J. Liotta, Phys. Rev. C61 (2000) 061301(R).

12. Y.R. Shimizu and K. Matsuyanagi, Prog. Theo. Phys. 72 (1984) 1017; T. Nakatsukasa, S. Mizutori, and K. Matsuyanagi, Prog. Theo. Phys. 87 (1992) 607.

13. D. Zawischa, J. Speth, and D. Pal, Nucl. Phys. A311 (1978) 445.

14. K. Hagino and H. Sagawa, Nucl. Phys. A695 (2001) 82.

15. K. Hagino and Nguyen Van Giai, e-print: nucl-th/0305034.

16. B.R. Johnson, J. Chem. Phys. 69 (1978) 4678.

17. D.J. Rowe, Nuclear Collective Motion, Methuen, London, 1968.

18. Nguyen Van Giai, Ch. Stoyanov, and V.V. Voronov, Phys. Rev. C57 (1998) 1204.

19. A. Bohr and B.R. Mottelson, Nuclear Structure Vol. 1, Benjamin, New York, 1969.

20. J. Terasaki, H. Flocard, P.-H. Heenen, and P. Bonche, Nucl. Phys. A621 (1997) 706.

21. M. Beiner, H. Flocard, N. Van Giai, and P. Quentin, Nucl. Phys. A238 (1975) 29.

22. D.H. Youngblood, Y.-W. Lui, and H.L. Clark, Phys. Rev. C60 (1999) 067302, and references therein.

23. I. Hamamoto, H. Sagawa, and X.Z. Zhang, Phys. Rev. C55 (1997) 2361.

24. S. Shlomo and G.F. Bertsch, Nucl. Phys. A243 (1975) 507.

25. Z.H. Levine and P. Soven, Phys. Rev. A29 (1984) 625.

26. T. Nakatsukasa and K. Yabana, J. Chem. Phys. 114 (2001) 2550. 


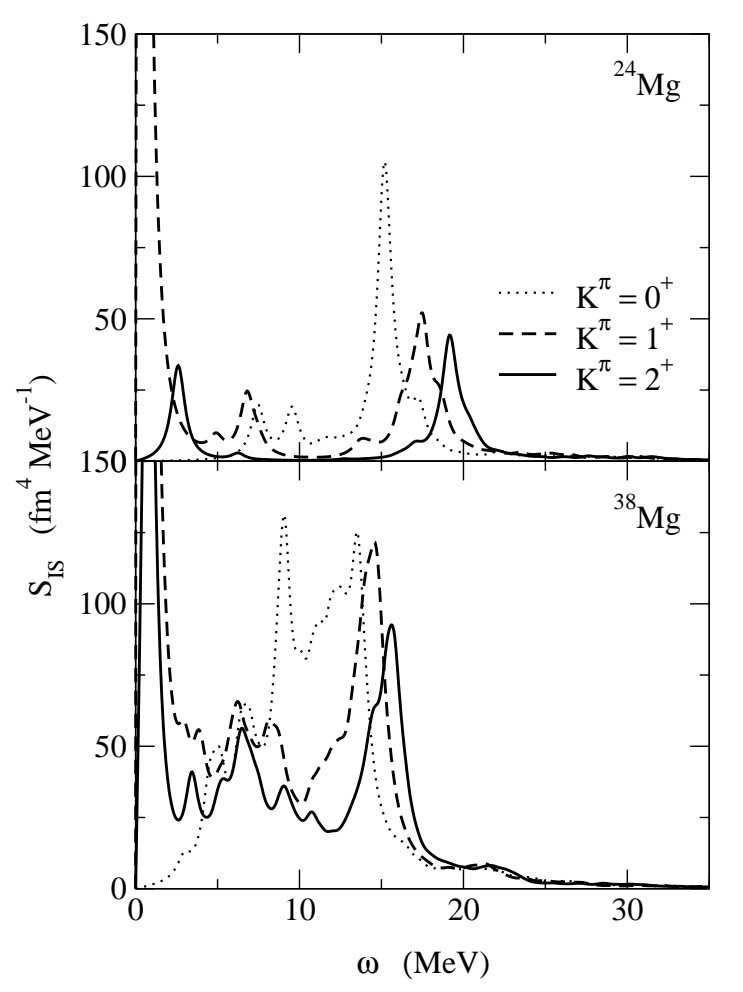

Figure 1. The QRPA responses of the ${ }^{24} \mathrm{Mg}$ nucleus (the upper panel) and of the ${ }^{38} \mathrm{Mg}$ nucleus (the lower panel) to an isoscalar quadrupole field. The dotted, dashed, and solid lines are for the $K^{\pi}=$ $0^{+}, 1^{+}$, and $2^{+}$modes, respectively.

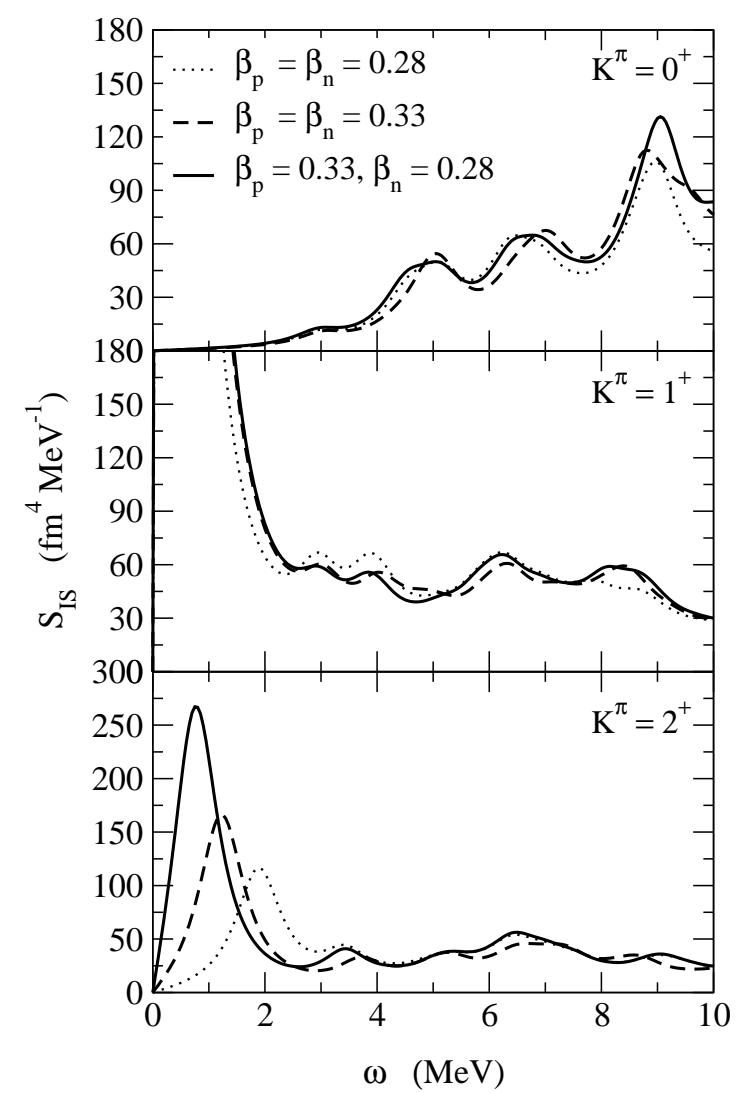

Figure 2. The effect of different proton and neutron deformations on the isoscalar quadrupole response of the ${ }^{38} \mathrm{Mg}$ nucleus. The dotted and the dashed lines are obtained by setting the proton and the neutron deformations to be the same as indicated in the inset, while the solid line is the result when the proton deformation is larger than the neutron deformation by 0.05. The top, middle, and bottom panels are for the $K^{\pi}=0^{+}, 1^{+}$, and $2^{+}$modes, respectively. 